\title{
DOS CASOS DE ESCLERODERMIA GENERALIZADA EN LA INFANCIA
}

\section{Prof. ADALBERTO STEEGER y Drs. AMANDA FRITIS y J, DANERI NOVOA Cátedra de Pediacria del Prof. A. Steeger. Hospital Manuel Arriarán.}

Nos permitimos relatar dos casos de esclerodermia generalizada observados en el Hospital Manuel Arriarán.

Si bien no se conoce su etiología y su tratamiento específico, es una entidad clínica bien definida y que debe ser tomada en cuenta y no olvidada por el pediatra.

$\mathrm{La}$ esclerodermia en la infancia se observa con escasa frecuencia y se caracteriza clínicamente por un endurecimiento coriáceo o alteración edematosa infiltrativa de los tegumentos que en ocasiones termina en una atrofia de la piel $y$, anátomo-patológicamente, por un cuadro característico y uniforme.

Se le conoce desde hace tiempo. Zacuto (1634) tiene una descripción completa de esta enfermedad. Luego con una serie de trabajos se han distinguido tres formas de esta enfermedad, sin contar el esclerema neo matorum $\mathrm{y}$ otros procesos que se han descrito como entidades apartes y desde 1903 con Ehrman, es considerada como una enfermedad general, al describir las lesiones esofágicas ${ }^{4}$.

CASO N? 1. - Obs. 79422. R. A. V.

Niño de 4 años, hijo de padres jótuenes y sanos. que sólo acusa coqueluche a tos 2 años.

Consulta el Policlinico por presentar desde hace un mes, edema duro de la cara, cuello y dorso que poco despucs se ha extendido a toda la mitad superior del cuerpo.

Al examen físico. se aprecia un niño de 4 años que pesa 15 kilos y con talla de $1.05 \mathrm{mts}$. que presenta color pálido amarillento de la piel de todo el cuetpo en la que se obscrva un edema duro de la cabeza a los pies: este empastamiento edematoso es mucho más manifiesto en la cara, cuello, nuca y hombros y región pectoral, to que le impide hacer movimientos de rotación y flexión de la cabeza. La piel aparece engrosada, brillante, (los surcos normales están borrados) y sobre todo consistente 7 duta siendo imposible levantar an pliegue de la misma. El rostro está rígido e iatnóvil (cara de máscara), la mímica abolida, la boea se aptecia pequeña, los párpados no pueden cetrarse completamente, la nariz está puntiaguda y adelgazada.

Este empastamiento edematoso es mucho menos manifiesto en la piel del abdomer. escroto y pene.
Se le practicar diversos exámenes con Jos que se descarts la lúes y tbc. Colesterinemia 4,10\%. Reserva alcalina 43,9 vol. \%. Electrocardiograma normal. Radiografía de silla turca (parte ósea notmal). No hay alteraciones de su contenido.

Biopsia de la piel: en la superficie del corte se reconoce la epidermis de aspecto normal. Las papilas y anexos de la piel normales. Dermis de grosor normal. llamando la atención sólo un intenso edema que dísocia las asas de las fibras colágenas. Diagnóstico: Esclerodermia.

Después de una estada en Argentina de 6 meses. vuelve a Chile y las lesiones empiezan a regresar rápidamente, primeto las de ta mitad inferior del cuerpo y más lentamente las de la mitad superior.

Al cabo de un año $y$ sin mediar tratamiento al guno las lesiones de la piel Itegan a restituirse ad integrum. No bubo compromiso orgúnico manifiesto lo que se corrobora con los exámenes de laboratotio.

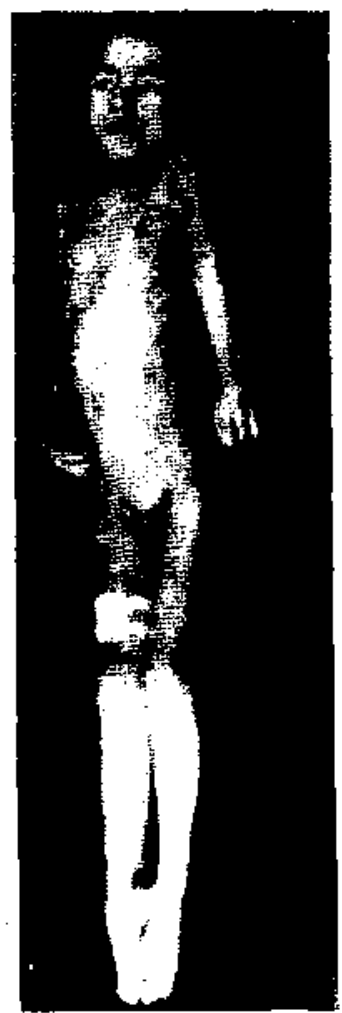

H. C. Caso No 2 
CASO N? 2. - (Ohs. clinica 115654). H. C. C.

Se trata de 1 niño de 6 años y medio de edad. Sin antecedentes hereditarios ni personales de imporfancia. Vacunaciones: antivariólicas en 2 ocasiones. B. C. G. al racer y antítifica (hace 2 años).

Enfednedad actual: a la edad de $51 / 2$ años y hace un año attás comenzó con nn critema de la cara. rodillas, codos y dorso de las manos, aproximadamente de 8 dias de duración y gue motivó un falso diagnóstico de sarampión, por to tual debió permanecer en cama. Posteriormente y sin precisar cuanto tiempo después de en baño en agua fria, comenzó en forma insidiosa con decaimiento. inapetencia $y$ diaforesis abundante, fiebre alta y poro a poro apareció un espastamiento progresivo de la piel del brazo derecho, luego del izquierdo $y$ después del tórax y cara, sin impedir alteraciones notorias de la motilidad. En es. tas condiciones es hospitalizado en los Andes; los sintomas clinicos más destacados los condensa el médico tratante: 1) facie rígica y ligero trismus; 2) toda la piel gruesa $y$ empastađa $y$ abundante transpiración generalizata: 3) edema de manos y pies; 4) moderada parálisis espástica de los miembros inferiores y leve de los superiores que sólo dan una ligera límitación de los movimientos que, en un comienzo le petmitían alimentarse con dificultad.

Se practican algunos exámenes: 1) orita: alb. $0.10 \mathrm{gr}$., uno que otro glóbulo rojo; 2) sangre: uremia0 0.45. Sedimentación $22 \mathrm{~mm}:$ 3) L.C.R. clato, a ligera presión, con glucosa 0.75 g. \%. Clorutos 7 g. 'Tr. Albúmina: no hay. Sin gérmenes.

En los primeros meses de haspitalización ef cua. dro es casi estacionario: en los últimos meses aumentta la atrofia de los músculos de los brazos y maslos con aparición de deformación en equipo de ambos pies. Se trató con Aurcomicina y Vit. $B_{1}$ sin prec'sar dosis. Sólo se presentó una otitis media supurada bilateral que duró una semata. como enfermedad intercurrente, sin mayor repercusión ostensible sobre su estado general. Al 42 mes. junto a ta atrofia muscu. lar. se peesentó edema de todo el cuerpo y cara la deglución se hizo dificultosa. (Datos dados por la madre\}.

Es enviado a Santiago con los diagnósticos probables: ¿mielitis post. sarampión? ¿Poliomialitis de evolución atípica? En nuestro setvicio, se recibe, después de 9 meses de enfermedad. a un niño de $63 / 2$ años con $20 \mathrm{kls}$. de peso, talla aproximada a $111 \mathrm{cms}$. Impresiona la posición pasiva con psiquis lúcido, pero cara de máscara, inexpresiva, estado de indiferencia $Y$ la rigidez de todos los músculos del cuerpo; además tiene una dificultad en la articulación de las palabras: đeglute bien.

La cara de máscara es pálida con discreto edema palpebral, desaparición de los pliegues pormales, edema duro del resto que impide hacet el pliegue; in transpiración es intensa y permanente. Dormía con los ojos entteabiertos por la dificultad para cerrar los pár. pados. hoca pequeña: orejas normates. la lengua conserva sus movimientos normales.

Cuello y columna: rigidez discreta de la naca: piel levemente infiltrada. Columna vert. con tígidez dularosa, a los movimientos pasivos. Tórax: limitación de la excursión toráxica, lo que se manifiesta por un tipo de respiración superficial; no presentaba disnea.

La piel presentaba los caracteres ya enunciados, más acentuados en la región dorsal y lumbar.

Pulmón $y$ corazón normales. Movimientos respiratorios 24. Pulso 102. Presión 10/8. Abdomen: algo abultado, con discreto meteotismo. La piel aparentemente infilstada pero en menor grado en el tórax. Hay una disminución discreta de la acción de los músculos abdominates $y$ no bay incontinencia de la orin.a y materias fecales. Sin embargo estas funciones se hacen con cierta dificultad inicial.

Higado, bazo y ombligo normales.

Genitales: edema del prepucio. Pie1 del pene $y$ bolsas normalcs.

Extremidades: la infiltración edematosa era tháxima en ellas. más marcada en las extremidades inferiotes que se presentaban como unos cilindros que semejaban una pata de mesa. Hiperhidrosis. Era imposible doblar las piertias y los brazos y aún los dedos por la lesión de la piel y la contractura muscular.

Mano izquierda: en el dorso presenta una cicatriz viciosa del tamaño de 1 peso, dura adberida. doloross, sin alteración de st coloración.

Pies: posición irreductible de equinismo varas.

Signos negativos: i) sin cianosis, sin alteración de la pigmentación de la piel; fanéreos (pelos, uñas), notmales:

2) sensibilidad tactil, dolorosi térmica y profunda conservadas;

$3)$ indempidad de los nervios eraneanos:

4) reflejos alterados por la rigidez.

Exámenes de faboratorio: Podemos resumirlos en los siguientes puntos:

1. - Investigación TBC: Mantoux (-) , Radioscopias tórax ( - ) Sedimentación: $8 \mathrm{~mm}$.

II. - Investigación Lúrs: Kahn (--) .

III. -- Invesrigación Neurológica: L.C.R. (-), cultivos (-). Electro-cncetalograma y electromiograma: normales. Examen psicológico: Torpeza simple. Fondo de ojo: normal.

IV. - Investigación Radiológica: carpo $\mathrm{y}$ rodi11a: ostcoporosis. Columna dorsat: normal. Columna lumbur: espina bifida de la $2^{3}$ sacra. Ambos hombros: osteoporosis y atrofia del himero. con elevación de la cabeza derecha. Ambas manos: osteoporosis $y$ atrofia del ssqueleto. Posición en flexión de los dedos. Sombra cáléca de la mano izquicrta. Ambos pies: ostroporosis del esqueleto. Varus.

v. - Otros exámenes: orina y deposiciones: not. males. Electrocardiograma: normales.

VI. - Investigación sanguínea: a) Celular. Rojos: término medio 4,8 millones. Blancos, térnino 


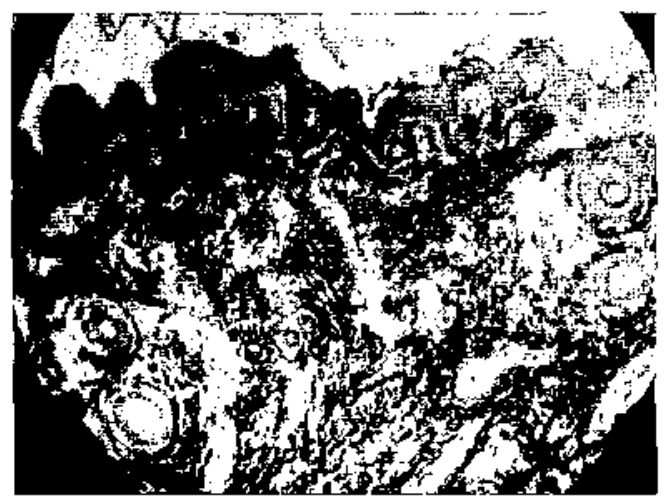

Mictofotografia. Piel.

$x$ 90. Hematox-Eosina. Discreta atrofía de la cpidermis. Dermis engrosado formado de manojos de tejido conjuntivo fibroso.

medio, 7 mil. Hemoglobina término medio, $88 \%$. Bac. término medio 9. Seg. término medio 49. Linf. término medio 28. Eos. término medio 0.5. Caracteres morfológicos: normales. Sangre perifética: no se observan células ni otros fenómenos de LE cells.

b) Quimica: uremia y glicemia: notmales: cakemí $12,4 \mathrm{mg} \%$ : fosfermis $4.5 \mathrm{mg}$. \%: proteinemia 72 gr. \%: (A.lb. 38. Glob. 27) : cloremia globalar $2.00 \mathrm{gr}$. ": tr: cloremia plasmática $3.72 \mathrm{gr.Mc}$ p pruebas hepáticas: bilirrubinemia total $5 \mathrm{mg}$. \%: bilitruminemia inđitecta $5 \mathrm{mg}$. \%. floculación: $(++)$.

VII. - Biopsía. Piel y múscalo; Discreta atrofia de la epidertis con el dermis engrosado. constituido por aptetados manojos de tejido conjuntivo fibroso. Fil trozo de músculo está formado por delgados manojos mesculares rodeados de tejido fibroso y adiposo. Se hace el diagnóstico de esclerodermia. Atrofia muscular. (Dr. J. Espinaza).

Diagnéstico. - Ante el cuadro clínico que presentaba este enfermo con los signos y síntomas ya enunciados se pensó en tha enfermedad del colágeno el cual se confirmó con el examen histológico considerándolo como un dermato esclero miositosis generalizada con compromiso visceral.

Diagnóstico diferencial: se puede desechar el diagnóstico de mielitis ya que no presenta alteraciones de la sensibilidad ni hay alteraciones de los esfinteres y la arreflexía que presenta se puede explicar por altetación de los tegumentos. Se paede desechar el diagnóstico de polionielitis por el L.C.R.. indemnidad de los nervios craneanos $y$ por el estado de las extremidades.

Mixedema. - En la infancia es frecuente y se acompaña a los cuadros de bipotiroidismo con todo su compléjo sintomático; sólo puede confundirse con el periodo edematoso de la esclerodetmia.

Enfetmedad de Addison. - La pigmentación es más localizada en la esclerodermia y se presenta con mayor frecuencia en la forma circunscrita $y$ en la forma progresiva.
Lepra nerviosa. - Las Jesiones son mís localizadas y predominan los fenómenos anestésicos que están ausentes en la escietodermia:

Reumatismo deformante. - Las deformaciones aue se producen en las extremidades no resultan del estado de la piel. la que es normal y movible.

Siringomelia. - No hay cscletosis y existe disociación de las sensibilidades.

Cuadros neturoloupicos con espassicidud. - En ellos hay signos de compromiso del neurón central y no existen altericiones cdematosas, indurativas $y$ atróficas de la piel.

Evolucion: et cuadro que pecsentaba este niño se mantuvo en forma estacionaria, con peq̨ueñas febricu. las irregulares hasta que se inició cl tratamiento con cortisona.

Para comenzar este tratamiento se hizo una prueba de Thorn (cortisona que fué positiva de $167 \mathrm{cosi}-$ nófilos a $4.7 \times \mathrm{mm}^{3}$ ) : se instituyó un régimen sin sal y se reguló la ingestión de liquidos con medición di la diuresís, además se agregó cloruro de potasio 1.20 gr. al dia y cloruro de amonio en igual cantidad. presentando hasta la fecha buena tolerancia.

Se controló, además, la presión arterial djariamente $y$ el peso cida dos o tres días debido a la dificultad de la actitud que presentaba el paciente.

Dosis: se inició el tratamiento con $100 \mathrm{mgts}$. diarios intramuscular, en dos dosis por 5 días y luego se continuó con 50 mgrs. diatios hasta compietar 850 mgrs. Y scgnir con una dosis de mantención de 50 mges. dia por medio. Total: 1,5 grs. Luego de una semana y media de descanso se instituyó tratamiento con $\mathrm{ACTH}$ : 40 migrs. $x 4$ días y luego 20 migrs. con dosis de mantención de 20 migrs. $x$ cada 3 dias. dia por medio, hasta completar $280 \mathrm{~m}$ Igrs. y seguir con dosis de mantención de 20 mlgrs. $x$ cada 3 dias.

Resultados. - $1^{\circ}$ Clinico: durante los primeros cuatro dias no se notó ringuna alteración del euadro clinico salvo una baja de peso de 800 grs. debido a que el niño no toleraba este nuevo régimen alimenticio. La diuresis se mantuvo alrrededor de los 400 grs. diarios: el $5^{\circ}$ dia presentó una alza de la presión a 162/118 que esa misma tarde (20 hrs.) bajó a $100 / 87$.

Ya desde el $6^{\circ}$ y 70 día se aprecia una gran mejoria, haciéndose la piet más elástica. permitiendo hacer pliegues al principio dificilmente y luego con cierta facilidad. I_a infittración disminuyó apreciablemente. La cata, aunque siempre pâlida, tomó tasgos de enra rormal. pudo cerrar los párdados petfectamente. En el cuello disminuyó la rigidez, y algo menos en la columna vertebral.

En las extremidades se aprecia mayor soltura en los limitados movimicntos de los brazos $y$ algo más amplios, en los antebrazos y manos, permitiendo tomar algunas cosas $y$ jugar en la cama.

Las sudoraciones ban disminuido, notándose al comienzo crisis cortas en las tardes. Las sensaciones 
subjetivas de dolores y frío a lis piernas han desapjrecido $y$ el niño se aprecia eufórico.

$2^{\circ}$ Combios de laboratorio. - Test de Thorn antes del tratamiento de cortisona $\mathrm{y}$ antes del ACTH, fueron positivos.

Todos los otros controles hasta la fecha están dentro de los limites notmales, satvo las pruebas de floculación que se mantienen en $(++) y(+t+)$.

Las esclerodermias en la infancia se observan con escasa frecuencia y se caracterizan: 1) clínicamente por un endurecimiento coriáceo o alteración edematosa infiltrativa de los ligamentos, que habitualmente termina en una atrofia de Ia piel $y ; 2$ ) anátomo-patológicamente nor un cuadro característico y uniforme.

Las diversas clasificaciones de esta afección se reúnen hoy día en dos entidades bien definidas: 1 ) las esclerodermias verdaderas o propiamente tales, en sus formas circunscritas y generalizadas. En ellas se ha incluído la esclerosis de Buschke que corresponde sólo a la etapa edematosa de la esclerodermia generalizada. 2) los cuadros pseudos esclerodérmicos: la adiponecrosis y el escleredema del recién nacido: a) la ađiponecrosis se presenta en el R. $N$. eutrófico o con exceso de peso y que han tenido un parto difícil. En los primeros días aparecen induraciones de la piel del tamaño de una arveja hasta el porte de la palma de la mano localizada en las mejillas, región glútea, parte externa del muslo, espalda, hombro y brazo. La piel a este nivel es lisa o bien como cáscara de naranja, la depresión con el dedo no deja huellas y no se puede hacer pliegues. La afección regresa lentamente sin dejar rastros y no se acompaña de signos generales (alteración de peso, diarreas, fiebres, diuresis, etc.). La anatomía patológica se caracteriza por una destrucción de células grasas, aparición de agujas cristalinas y de tejido proliferativo fibroconectivo en el que se puede depositar ocasionalmente el Ca. b) el esclerema del R. N. que se presenta en los prematuros, débiles, aparece en los primeros días de vida con hipotonía o procesos infecciosos graves.

Se distinguen dos tipos a) el escleroedema o esclerosis edematosa y b) el esclerema graso o esclerema sencillamente; el esclerema del adulto e infantil de Buschke que era considerado como una entidad aparte, hoy día se encuadra en el primer período o período edematoso de la esclerodermia verdadera generalizada que evoluciona en 3 períodos: p. edematoso, p. de induración y p. escleroso atrófico o restitution ad integrum.

Las esclerodermias verdaderas se dividen en: circunscritas y generalizadas.

Las formas circunscritas se presentan como placas (morohea) o en bandas (esclerodermia en banda o en golpe de sable) o bien en forma parcial (mitad de Ja cara o del cuello etc.).

Las placas son al comienzo aisladas; la piel tiene un tono lívido rojo en cuyo centro aparecen manchas o rayas decoloreadas que aumentan con el tiempo.

La piel es dura como cuero, blanda, de tinte amarillo verdoso, marfil o de cera rodeado de un anillo azuI violáceo. Después se puede pigmentar la piel o quedar como un vitiligo, la dureza de ésta disminuye dejando una piel blanda atrófica semejante a una cicatriz de color pardo. Otras variante de esta enfermedad son la White spot disease o dermatitis liquinoides atrófica y la esclerodermia de Unna (en forma de tarjeta). La A. P. de éstas es característica y uniforme para la esclerodermia verdádera.

Las esclerodermias localizadas a veces regresan espontáneamente $\mathrm{y}$ otras veces se generalizan.

Esclerodermias difusas. - Como su nombre lo indica el proceso es difuso y ataca no solo el tejido conjuntivo de la piel, músculos, huesos, sino el de muchos órganos dando una sintomatología multiforme de acuerdo con los órganos afectados (hígado. riñón, bazo, pulmón, corazón. etc.). Se deseriben dos formas: a) esclerodermia generalizada aue presenta un periodo edematoso (o ésclerosis de Buschke), que afecta todo el cuerpo y poco a noco se hace indurado (esclerodermia indurada) y que toma también Ios músculos estriados y a veces los huesos. Otras veces afecta los músculos respiratorios $\mathrm{v}$ de la deglución (como en el caso 2). Este estado indurativo puede seguir dos caminos o a la vestitution ad integrum (caso N? 1) o bien a Ia atrofia o esclerosis (período atrófico esclerótico); b) la esclerodermia difusa progresiva o esclerodactilia. que comienza en los dedos, cara, quedando un tiempo estacionaria $\mathrm{y}$ luego se generaliza. Su comienzo se caracteriza por alteración del estado general, falta de movimientos, dolores y parestesias $v$ A veces como un síndrome de Raynaud, el 
estado edematoso es muy fugaz para Iuego pasar al período indurativo, la piel se hace muy vulnerable $y$ por pequeños traumas da lugar a úlceras, las uñas se deforman y se atrofian.

E1 resto de la musculatura se toma al igual que las sinoviales, articulación y huesos $\overline{2} \cdot 4 \cdot 6 \cdot 12 \cdot 13 \cdot 15 \cdot 16 \cdot 19 \cdot 20.24 .25$.

EI primer caso se trata de un niño que tiene una esclerodermia casi generalizada en un periodo edematcso indurativo; sin alteración de otros sistemas orcánicos y que regresa espontáneamente. El $2^{2}$ caso se refiere a una afección generalizada en un período indurativo atrófico sin compromiso de otros sistemas orgánicos y cuya evolución está modificada por el tratamiento.

La anatomía patológica de la esclerodermia generalizada se caracteriza por degeneración fibrinoide y proliferación de células epiteliales.

1) La deg. fibrinoide corresponde: a) sustancia fundamental que sufre una serie de alt. físico-químico dando lugar a una substancia homogénea de alto índice de refractibilidad $y$ marcada apetencia tintorial eosinófila; se le puede comparar a una gelatina, y esta alteración se manifiesta clínicamente por la infiltración edematosa de la piel (estado edematoso); b) las fibras colágenas se separan por la alteración antedicha de la sust. fundamental y sufren también la degeneractón fibrinoide. degeneración que ataca también al teiido conectivo que rodea los vasos sanguíneos $\mathrm{y}$ aun las fibras musculares con obstrucción del lumen.

Si esta lesión vascular se propaga a los tejidos conectivos de los diversos órganos aparecen signos clínicos dependientes de la alteración funcional del órgano correspondiente.

La degeneración constituye el primer período de la esclerodermia.

2) I a proliferación de células reticulares fibrocitos, y endoteliales de los vasos sanguíneos e infiltración de histiocitos, plasmazellen y linfocitos del tejido conectivo constituve la $2^{\mathrm{a}}$ etapa del proceso anátomo-patológico de esta afección Y se traduce clínicamente por el período de induración.

En último término hay esclerosis total con reemplazo re los elementos citológi$\cos$ por neutrófilos $\mathrm{v}$ eosinófilos (período escleroso atrófico) 3.5.8-9-14.21.

Muchas son las teorías que tratan de explicar estos hechos anátomo-patológicos porque no se conoce bien la etiología ni la patogenia de la enfermedad.

La persistencia de la calidad normal de la substancia fundamental del tejido conectivo se debe a un equilibrio de los ácidos mucopolisacáridos: el ácido hialurónico, condroítico, y condroitin sulfúrico. Si se agrega a esta substancia principal una proteína alcalina el ph del tejido cae por debajo del punto isoeléctrico de sus proteínas por precipitación de los mucupolisacáridos, se forma así una substancia nueva fíbrinoide responsable de la esclercsis. Las proteinas alcalinas serian liberadas en el teiido conjuntivo, en el sitio de la lesión, por un agente causal, hasta aquí desconocido.

Las diversas etapas de la A. P. de Ia esclerodermia, que es similar a la A. P. de otras entidades clínicas como el lupus eritematoso generalizado. la dermatomiositis, artritis reumatoidea. periarteritis nudosa, enfermedad reumática, tromboarteritis obliterante, etc., han llevado a los clínicos y anátomo-patólogos a crear una concepción unitaria de todos estos cuadros incluyéndolos en las enfermentades del colágeno.

Las ideas de Selye han afirmado más esta posición unitaria actual.

\section{Agentes etiológicos:}

a) Factores predisponentes. I. -- Se habla de la constitución especial de estos enfermos. En las dos cbservaciones qua presentamos no podemos afirmar una constitución especial ni comprobar antecedentes de diátesis alguna.

II. - Las carencias nutritivas contribuirían al estallido de la enfermedad: en los dos enfermos hay francos antecedentes de carencia proteica y grasas.

III. - Se habla vagamente sobre alteraciones endocrinas que nadie las define en forma precisa.

b) Factores desencadenantes. Se señala el frío, traumatismo, fatiga, shock físico, infecciones, etc. En el primer niño yo se registra ninguno de estos factores: en el $2^{\text {? }}$ se anota el comienzo de la enfermedad con un baño frío prolongado.

Estos diversos factores provocarían, en un terreno determinado, una descarga de hormonas suprarrenales o hipofisiarias semejante al periodo de alarma de Selye 1-10-11-13-22. 
La comprobación de estos hechos ha llevado a los clínicos a emplear los glucocorticoides (la cortisona) o las hormonas corticotropa hipofisiaria en el trotamiento de estas afecciones, lo que se hizo solamente en el $2^{\circ}$ caso ya que en el $1^{\circ}$ no se consiguió la droga y la regresión del cuadro fué espontáneo.

Tratamiento. - Se han ensayado diversos tratamientos, vit. C, ergosterol, bismuto sódico, priscol, vacuna tífica, hormonas sexuales, ácido para-aminobenzojco, con resultados dudosos, la difenihidramina, glucosifona, etc.

Ultimamente se ha ensayado la simpactectomía con resultados muy inciertos.

La paratiroidectomía es menos recomendable aue el tratamiento enunciado anteriormente por los peligros postoperatorios.

El tratamiento con novocaína endovenosa (solución al $1 / 1.000$ ) empezando con dosis de $\mathbf{5}$ cc. inyectable muy lentamente. hasta alcanzar 10 a 15 cc. parece haber sido eficaz en algunos casos ( 2 a 7 casos tratados por el Dr. Cristian Barría).

La vitamina $\mathbf{E}$ en dosis de 200 miers. diarios parece haber tenido éxito en algunos casos de esclerodermia circunscrita.

Tratamiento con ACTH y cortisona. - Se basa en: 1) la acción a antihialuronidasa y antifibroplástica.

2) Estimula el metabolismo celular.

3 ) Posibles efectos sobre centros diencefálicos. En contra tenemos la tendencia a la alcalosis y la baja de potasio que alteraría el metabolismo celular y la permeabilidad de las membranas.

Mientras se ejerce el tratamiento. aumenta el apetito, desaparecen los dolores y la disfagia, $y$ se recuperan algunos movimientos, pero, al suspenderlo, los síntomas se hacen nuevamente presente ${ }^{7.17}$. 23.26 .

\section{RESUMEN}

Se presentan dos casos de esclerodermia generalizada en niños de 4 y 6 años. En el primero, sin compromiso visceral, las lesiones regresan espontáneamente sin tratamiento alguno $\mathrm{y}$, en el segundo, en que hay compromiso muscular, óseo $y$ visceral, ésta está actualmente en tratamiento con ACTH, después de una cura de 1.50 grs. de cortisona.

\section{SUMMEARY}

The authors report on two cases of generalized sclerodermia in children aged 4 and 6 years. In the first case, who had no visceral lesions, the skin manifestations regressed spontaneously, while the second case with muscular. osseous and visceral involvement is still under treatment with ACTH, after receiving $\mathbf{1 , 5 0}$ grs. of Cortisone.

\section{BIBLIOGRAFÍA}

1.-ACEVEDO, VETASCO - MTNNTCIT - Ese'eniler-

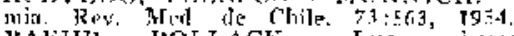

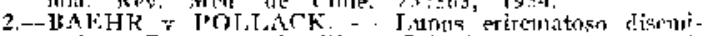

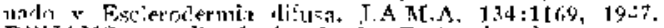

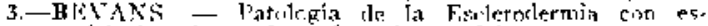
ret:ial futerés a las alterationes del aporato gastrointestitul. Anl. T. I'ath. $21: 35$. 1945.

A.-COSTE, BOCREI, CIFATTL y JACROXIOYIE.

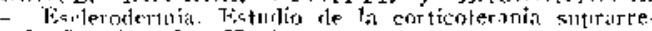
rol Semaine did Fofititux, $28: 176$ \$ 1952 .

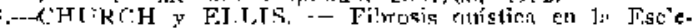

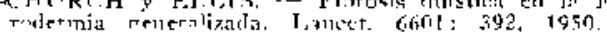

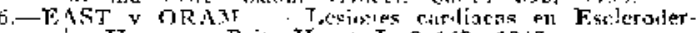

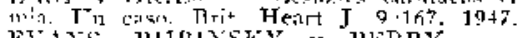

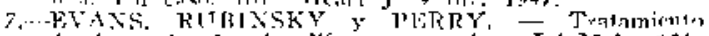
tle lis pascowdermia difusa jengresiva. J.A.M.A. 151:

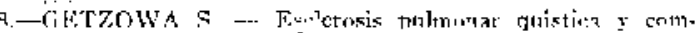

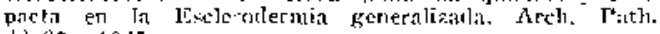
Ail:00 1255

9. -HABVTKR - RANMTET.F. Eeceredterinia tirn-

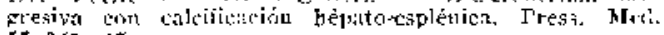

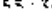

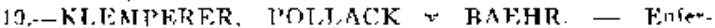

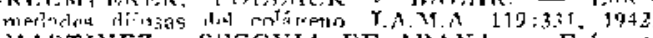

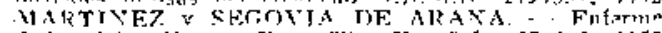

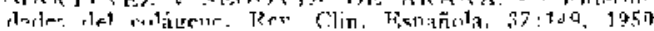

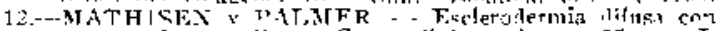
rimlintrinitan cardiacr. Cakn clinico. Atwer. Heart, J

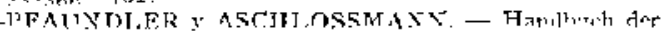

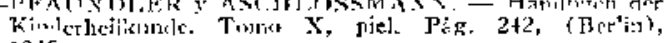
19.35

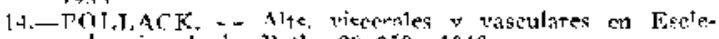

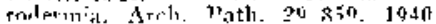

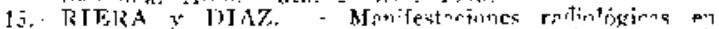

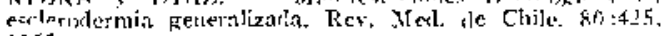
1059

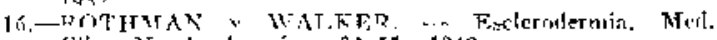
Pin

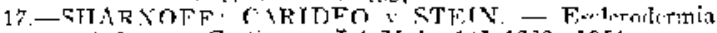

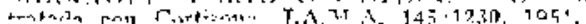

18 -

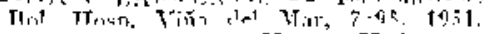

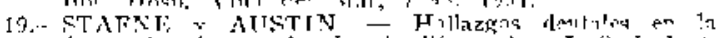

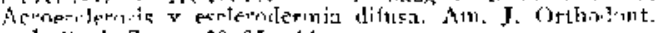

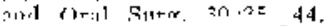

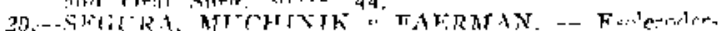

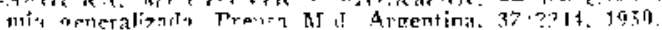
Sएव

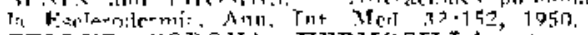

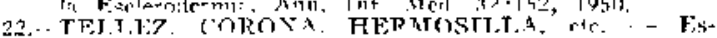

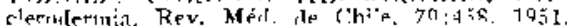

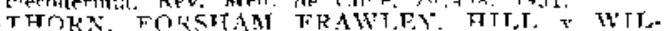

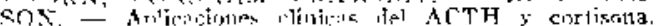

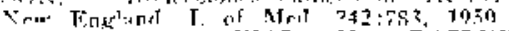

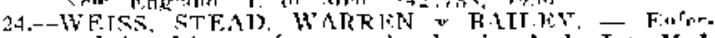

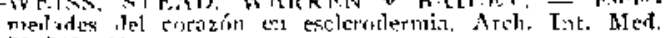
$71: 749.43$.

25, - WFISSEIRACT, STTWWART - HOLST.T - A'teraciones esificiris eu la escterotermia. Ant. ite Jermat. $9: 81.1038$

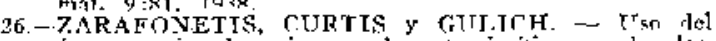

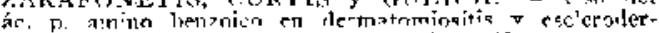
mia. 6 casas. Arzh. Int. Med. $85: 27,1950$. 